\title{
Screening for Oral Cavity Cancer: A 1-year Experience of a Regional Hospital in Taiwan
}

\author{
Tzu-Hang Chi, ${ }^{1,2}$ Chien-Han Yuan, ${ }^{1}$ Rong-Feng Chen' \\ ' Department of Otolaryngology, Kaohsiung Armed Forces General Hospital, Kaohsiung, Taiwan, ${ }^{2}$ Department of \\ Otolaryngology, Taoyuan Armed Forces General Hospital, Taoyuan, Taiwan.
}

\section{ABSTRACT}

Introduction: The purpose of this study was to analyze the risk factors affecting precancerous lesions, and cancer of oral cavity, and to assess efficacy of visual screening for oral mucosal lesions.

Methods: The medical records of patients older than 30 years of age with history of habitual cigarette smoking or betel quid chewing that received screening for oral mucosal lesions between January 2012 and December 2012 were retrospectively reviewed. The patients' age, gender, risk factors, screening findings, and histopathology results of biopsy were included for further analysis.

Results: A total of 1341 patients were enrolled in this study. There were 1080 males and 261

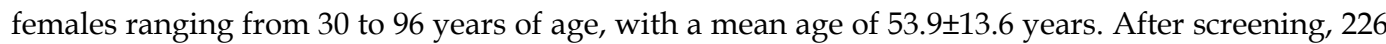
$(16.9 \%)$ were found to be positive of oral lesions. Among these 226 patients, 69 (30.5\%) underwent biopsy under local anesthesia, and the histopathology showed malignancy in 13 (5.8\%). All of the confirmed malignant cases were squamous cell carcinoma. Among them, 12 received further staging examination and one was lost to follow-up resulting in unknown stage. The early stage oral cavity cancer (stage I and II) accounted for $84.6 \%(11 / 13)$.

Conclusions: The detection rate of early stage oral cavity cancer in our study was reasonable. Therefore, visual screening for oral cavity cancer is recommended for patients with habitual cigarette smoking or betel quid chewing.

Keywords: betel quid chewing; cigarette smoking; oral cavity cancer; screening.

\section{INTRODUCTION}

Oral cancer including oral cavity cancer and oropharyngeal cancer is the sixth most common cancer in the world. $^{1}$ It is well recognized that oral cavity cancer is a serious and growing clinical problem which is not rarely observed during practice of otolaryngology. According to the literature, about 274300 cases occur worldwide annually, and two-thirds of the cases occur in the developing countries. ${ }^{2}$ Several risk factors such as cigarette smoking, betal quid chewing, alcohol drinking, immunosuppression, and human papillomavirus infection have been found to contribute to the development of oral cavity cancer .3 In Taiwan, oral cavity cancer has been one of the ten leading causes of cancer-related death since

Correspondence: Chien-Han Yuan, Department of Otolaryngology, Kaohsiung Armed Forces General Hospital, 2, Chung Cheng 1 st Road, Kaohsiung, 802, Taiwan, Republic of China. Email: cooljackychi@yam.com, Tel: +886-7-7494965. 
1982. ${ }^{4}$ Cigarette and betel quid are still common used psychoactive substances even though the government has been devoted to educating the general public about their risks associating with the oral cavity cancer. ${ }^{5}$ For the purpose of early detection, treatment and better survival of oral cavity cancer, Bureau of Health Promotion, Department of Health in Taiwan has carried out a screening program of oral mucosal lesions for adults older than 30 years of age with history of habitual cigarette smoking or betel quid chewing since January 2011. The aim of this study was to assess the effects of screening for oral mucosal lesions managed by otolaryngologists in a regional hospital.

\section{METHODS}

We conducted a retrospective review of the medical records of patients receiving screening for oral mucosal lesions at Kaohsiung Armed Forces General Hospital between January 2012 and December 2012. Criteria for inclusion in this study were age above 30 years of age, history of habitual cigarette smoking or betel quid chewing, and screening with adequate illumination, visual and palpation examination by otolaryngologists. Exclusion criteria were prior history of oral cavity cancer, screening by health care workers. Finally, a total of 1341 patients were enrolled in this study. The information was collected from the medical records of a regional teaching hospital in Taiwan. The factors included in the analysis were age, gender, risk factors, screening findings, and histopathology results of biopsy. The data were analyzed using the Chi-square test. A $p$ value $<0.05$ was considered to be statistically significant.

\section{RESULTS}

Of the 1341 patients who were older than 30 years of age with history of habitual cigarette smoking or betel quid chewing and received screening for oral mucosal lesions, there were 1080 males $(80.5 \%)$ and 261 females $(19.5 \%)$ with a male: female ratio of $4.14: 1$. The mean age was $53.9 \pm 13.6$, with a range of 30 to 96 years.

Positive screening of oral mucosal lesions were found in 226 patients (16.9\%); of these, 198 had leukoplakia $(86.7 \%), 12$ had submucous fibrosis $(5.3 \%), 9$ had erythroleukoplakia $(4.0 \%)$ and seven had erythroplakia (3.1\%). Analyses of gender, age, and risk factors are shown in Table 1. The pattern of distribution categorized by gender and by the risk factors was statistically significant $(p<0.05)$. With respect to the locations of the 226 precancerous lesions, the most affected anatomical site was buccal mucosa $(n=169 ; 74.8 \%)$, followed by tongue $(n=23 ; 10.2 \%)$, retromolar trigone $(n=20 ; 8.8 \%)$, palate $(n=6 ; 2.7 \%)$, gingiva $(n=5 ;$ $2.2 \%)$, floor of mouth $(n=2 ; 0.9 \%)$, and lips $(n=1$; $0.4 \%)$.

\begin{tabular}{|c|c|c|c|}
\hline & $\begin{array}{l}\text { All } \\
\text { cases, } \\
\mathrm{n}\end{array}$ & $\begin{array}{l}\text { Precancerous } \\
\text { lesions, n (\%) }\end{array}$ & $p$ value ${ }^{a}$ \\
\hline All & 1341 & 226 (16.9) & \\
\hline \multicolumn{4}{|l|}{ Gender } \\
\hline Male & 1080 & $210(19.4)$ & $0.000^{*}$ \\
\hline Female & 261 & $16(6.1)$ & \\
\hline \multicolumn{2}{|l|}{ Age (years) } & & 0.079 \\
\hline $30-39$ & 239 & $35(14.6)$ & \\
\hline $40-49$ & 259 & $50(19.3)$ & \\
\hline $50-59$ & 440 & $67(15.2)$ & \\
\hline $60-69$ & 250 & 54 (21.6) & \\
\hline$\geq 70$ & 153 & $20(13.1)$ & \\
\hline \multicolumn{2}{|l|}{ Risk factors } & & $0.000^{*}$ \\
\hline \multirow{2}{*}{$\begin{array}{l}\text { Cigarette } \\
\text { smoking } \\
\text { Betel nut } \\
\text { chewing }\end{array}$} & 883 & $85(9.6)$ & \\
\hline & 59 & $17(28.8)$ & \\
\hline $\begin{array}{l}\text { cigarette } \\
\text { smoking and } \\
\text { betel nut } \\
\text { chewing }\end{array}$ & 399 & $124(31.1)$ & \\
\hline a Chi-square & st, ${ }^{*} p<$ & .05 & \\
\hline
\end{tabular}

Only less than one third of the 226 patients with oral precancerous lesions agreed to undergo biopsy under local anesthesia (69 patients, 30.5\%) whereas the rest of 157 (69.5\%) refused biopsy. Histopathology results of the 69 biopsy cases were parakeratosis or hyperkeratosis in 42 (60.9\%), squamous papilloma in eight $(11.6 \%)$, lymphoid hyperplasia in six $(8.7 \%)$, and malignancy in $13(18.8 \%)$. All malignant cases were confirmed to be squamous cell carcinoma. Among these patients undergoing biopsy, 13 cases with squamous cell carcinoma in the oral cavity were found in nine out of the 61 patients with leukoplakia $(14.8 \%)$, three out of the four patients with erythroleukplakia $(75.0 \%)$, one out of the two patients with erythroplakia $(50.0 \%)$, and none out of the two patients with submucous fibrosis (0\%).

The demographic characteristics of the patients with oral cavity cancer are shown in Table 2 . We found that most of the patients with oral cavity cancer were aged 50 years or older (12 of $13,92.3 \%)$. Our analyses revealed that the pattern of distribution categorized by 
age and the risk factors was statistically significant $(\mathrm{p}<$ $0.05)$. Regarding to the locations of the 13 malignancy, the most affected anatomical site was tongue $(n=6$; $46.1 \%)$, followed by buccal mucosa $(n=3 ; 23.1 \%)$, retromolar trigone $(\mathrm{n}=2 ; 15.4 \%)$ and palate $(\mathrm{n}=2$; $15.4 \%)$.

\begin{tabular}{|c|c|c|c|}
\hline & $\begin{array}{l}\text { All } \\
\text { cases, } \mathrm{n}\end{array}$ & $\begin{array}{l}\text { Oral } \\
\text { cavity } \\
\text { cancer, } \\
\mathrm{n}(\%)\end{array}$ & $\begin{array}{l}\mathrm{p} \text { value } \\
\mathrm{a}\end{array}$ \\
\hline All & 1341 & $13(1.0)$ & \\
\hline Gender & & & 0.709 \\
\hline Male & 1080 & $11(1.0)$ & \\
\hline Female & 261 & $2(0.8)$ & \\
\hline Age (years) & & & $0.018^{*}$ \\
\hline $30-39$ & 239 & $1(0.4)$ & \\
\hline $40-49$ & 259 & $0(0)$ & \\
\hline $50-59$ & 440 & $4(0.9)$ & \\
\hline $60-69$ & 250 & $3(1.2)$ & \\
\hline$\geq 70$ & 153 & $5(3.3)$ & \\
\hline Risk factors & & & $0.027^{*}$ \\
\hline Cigarette smoking & 883 & $4(0.5)$ & \\
\hline $\begin{array}{l}\text { Betel nut chewing } \\
\text { Cigarette smoking } \\
\text { and betel nut } \\
\text { chewing } \\
{ }^{\text {a }} \text { Chi-square test, }{ }^{*}\end{array}$ & $\begin{array}{l}59 \\
399 \\
0.05\end{array}$ & $\begin{array}{l}1(1.7) \\
8(2.0)\end{array}$ & \\
\hline
\end{tabular}

Beside one patient lost to follow-up, 12 out of 13 patients with oral cavity cancer received further procedures such as contrast enhancement computed tomography of nasopharynx to neck, chest $\mathrm{x}$-ray, abdominal ultrasound, and whole body bone scan to characterize the stage of the cancer. It was found that 11 cases were at early stage $184.6 \%$ in the population of 13 , including six cases at stage $I$ and 5 cases at stage II), and one case were advanced at stage IV. In 2009, there were 4192 newly registered cases of oral cavity cancer in Taiwan according to Taiwan cancer registry database. ${ }^{6}$ Among them, 1907 were at early stage (19 with stage 0, 979 with stage I, and 909 with stage II), 1970 were at advanced stage (502 with stage III and 1468 with stage IV) and 315 with unknown stage. The early stage oral cavity cancer accounted for $45.5 \%(1907 / 4192)$ in all registered cases. Therefore, the percentage of the early stage oral cavity cancer detected by the screening program in the present study was significantly higher than that of Taiwan cancer registry database in 2009 ( $p<0.05$ ) (Table 3).

\begin{tabular}{|c|c|c|c|}
\hline \multicolumn{4}{|c|}{$\begin{array}{l}\text { Table 3. Comparison of tumor stage between } \\
\text { screening and Taiwan cancer registry database. }\end{array}$} \\
\hline \multirow{3}{*}{$\begin{array}{l}\text { Stage } \\
\text { Early stage }\end{array}$} & $\begin{array}{l}\text { Screening } \\
(n=13)\end{array}$ & $\begin{array}{l}\text { Database } \\
(\mathrm{n}=4192)\end{array}$ & $\begin{array}{l}\mathrm{p} \text { value } \\
\mathrm{a}\end{array}$ \\
\hline & n (\%) & n (\%) & \multirow[t]{3}{*}{$0.013^{*}$} \\
\hline & $11(84.6)$ & 1907 (45.5) & \\
\hline Stage 0 & $O(0)$ & $19(0.45)$ & \\
\hline Stage I & $6(46.1)$ & $979(23.35)$ & \\
\hline \multirow{3}{*}{$\begin{array}{l}\text { Stage II } \\
\text { Advanced } \\
\text { stage } \\
\text { Stage III }\end{array}$} & $5(38.5)$ & 909 (21.68) & \\
\hline & $1(7.7)$ & 1970 (47.0) & \\
\hline & $0(0)$ & 502 (11.98) & \\
\hline \multirow{2}{*}{$\begin{array}{l}\text { Stage IV } \\
\text { Unknown } \\
\text { stage }\end{array}$} & $1(7.7)$ & $\begin{array}{l}1468 \\
(35.02)\end{array}$ & \\
\hline & $1(7.7)$ & $315(7.51)$ & \\
\hline a Chi-square & st, ${ }^{*} p<0.05$ & & \\
\hline
\end{tabular}

\section{DISCUSSION}

The prevalence of oral precancerous lesions in our study was $16.9 \%(226 / 1341)$ which was obvious higher than the reported $5.2 \%$ by Chang et al. ${ }^{7}$ in 2011. The difference is likely attributed to the study design and distinct population selection. Our study was based on both male and female patients older than 30 years of age with history of risk factors, whereas the study by Chang et al. was based on male patients older than 18 years of age without considering risk factors. According to the report by Phookan et al. ${ }^{8}$ in 1998 , leukoplakia was the most frequent type of oral precancerous lesions found, and buccal mucosa was the most common lesion site in India. In the current study, leukoplakia (86.7\%) and buccal mucosa (74.8\%) were also the most frequent oral precancerous lesion and site respectively. Furthermore, the precancerous lesions were found in about one third of the patients with both habitual cigarette smoking and betel quid chewing $(31.1 \%)$ in this study.

Among the precancerous lesions of the oral cavity studied, erythroplakia had the highest malignant transformation rate of $90 \%$, in contrast to other precancerous lesions such as leukoplakia, submucous fibrosis, and lichen planus with the malignant transformation rates of 3.6$17.5 \%, 0.5-6 \%$, and $0.4-3.7 \%$ respectively. ${ }^{9}$ Our study showed that the malignant rates of erythroleukplakia and erythroplakia patients undergoing biopsy were as high as $75.0 \%(3 / 4)$ and $50.0 \%(1 / 2)$ respectively, and most of the patients with malignancy (9 out of 13) were found in leukoplakia patients undergoing biopsy (9/61, $14.8 \%)$. The strong relation between oral precancerous 
lesions and oral cavity cancer was found in this study obviously.

Our study indicated that the prevalence of oral cavity cancer was around $1.0 \%$ (13 of 1341$)$ in the population with habitual cigarette smoking or betel quid chewing, and the oral cavity cancer was detected in different ages older than 30 years, except the 40 to 49 age bracket. More than $90 \%$ of the patients with oral cavity cancer were aged 50 years or order $(12 / 13,92.3 \%)$. This finding was obviously higher than the $63.9 \%$ of oral cavity cancer occurring in the patients with ages of 50 years and above in a previous study. ${ }^{10}$ According to Ko et al, ${ }^{11}$ the patients with habitual cigarette smoking, betel quid chewing, and alcohol consumption had the highest risk of oral cancer, followed by those with both cigarette smoking and betel quid chewing, and those with both betel quid chewing and alcohol consumption. It was reported that cigarette smoking or betel quid chewing alone, has a similar level of risks to each other, and was a weak risk factor contributed to oral cavity cancer. ${ }^{12}$ Our findings also indicated that the prevalence of oral cavity cancer in the patients with habitual cigarette smoking and betel quid chewing was higher than that with habitual cigarette smoking or with betel quid chewing alone. The synergism of cigarette smoking and betel quid chewing on the incidence of oral cavity cancer was noted.

This study revealed that the tongue was the most common location of oral cavity cancer and followed by buccal mucosa. This is likely related to the irritation caused by cigarettes and betel quids to the tongue and buccal mucosa of cigarette smokers and betel quid chewers. For the early stage oral cavity cancer, the overall five years survival rate is more than $50 \%$, however, the overall five years survival rate is less than $40 \%$ for the advanced stages. ${ }^{13}$ The early stage oral cavity cancer among the total 13 malignant cases detected by the screening program in our study was as high as $84.6 \%$, and this finding was markedly higher than the result of $45.5 \%$ from Taiwan cancer registry database in 2009. This difference indicated that screening by conventional oral cavity examination of the population with high risks is effective to detect precancerous lesions or cancers of oral cavity. Even though the detection rate of early stage oral cavity cancer in our study was reasonable, there were nearly $70 \%$ of patients with oral precancerous lesions refusing further biopsy. This may be ascribed to patients' fear to biopsy, physicians' insufficient health education, and lack of knowledge about oral cavity cancer in the general population. Educating the public about the knowledge and risk of oral cavity cancer would be helpful to encourage the high risk population to enroll in the screening program. Early detection of oral cavity cancer or lesions may significantly increase the chances for successful treatment and thus elevate survival rate and lessen morbidity or mortality.

\section{CONCLUSIONS}

In the present study, most of the patients with oral cavity cancer were both habitual cigarette smokers and betel quid chewers, and more than $90 \%$ of the patients with oral cavity cancer were aged 50 years or older. Leukoplakia was the most frequent precancerous lesion, but erythroleukoplakia had the highest malignant rate. The tongue was the most common location of oral cavity cancer and followed by buccal mucosa. Through screening, chance of detecting oral cavity cancer early can be increased, proper and early treatment can be conducted and survival improvement can be achieved.

\section{ACKNOWLEDGEMENTS}

The authors thank Miss Chi-Wei Lin, Miss Shu-Yan Shi, Miss Jing-Ying Li, Miss Chu-Ying Wong, Miss Ya-Lun Liu, and Mr. Chi-Hua Lee, Research team of cancer screening, Kaohsiung Armed Forces General Hospital, Kaohsiung, Taiwan for assisting in the running of the study.

5. Wen $\mathrm{CP}$, Tsai SP, Cheng TY, et al. Uncovering the relation between betel quid chewing and cigarette smoking in Taiwan. Tob Control 2005;44:116-122.

6. Bureau of Health Promotion, Department of Health, the Executive Yuan, Taiwan. Cancer registry annual report, 2009, Taiwan. http://www.bhp.doh.gov.tw/BHPNet/ Web/Stat/StatisticsShow.aspx?No=201206210001. Accessed April 3, 2013 (Chinese)

7. Chang IH, Jiang RS, Wong YK, et al. Visual screening of oral cavity cancer in a male population: experience from a medical center. J Chin Med Assoc 2011;74:561-566.

4. Lee $\mathrm{CH}$, Ko YC, Huang HL, et al. The precancer risk of betel quid chewing, tobacco use and alcohol consumption in oral leukoplakia and oral submucous fibrosis in southern Taiwan. Br J Cancer 2003;88:366-372. 
8. Phookan J, Saikia KP. A clinicopathological study of the pre-malignant conditions of oral cavity. Indian J Otolaryngol Head Neck Surg 1998;50:246-249.

9. Antony G, Sreenivasan BS, Sunil S, et al. Potentially malignant disorders of oral cavity. OMPJ 2011;2:95-100.

10. Muhsen HJ, AL-Raheem YA. Oral tumors: clinicopathological study among patients attending ENT clinic in Baghdad. Al-Kindy Col Med J 2009;5:11-18.

11. Ko $\mathrm{YC}$, Huang $\mathrm{YL}$, Lee $\mathrm{CH}$, et al. Betel quid chewing, cigarette smoking and alcohol consumption related to oral cancer in Taiwan. J Oral Pathol Med 1995;24:450-453.
12. Lumukana R, King T. Smoking and chewing habits of oral cancer patients in the Solomon islands. Pac Health Dialog 2003;10:41-44.

13. Pugliano FA, PiccirilloJF, Zequeira MR, et al. Clinical-severity staging system for oral cavity cancer: five-year survival rates. Otolaryngol Head Neck Surg 1999;120:38-45. 